\title{
Does the use of cardiopulmonary resuscitation feedback devices improve the quality of chest compressions performed by doctors? A prospective, randomized, cross-over simulation study
}

\author{
Jolanta Majer ${ }^{1}$, Miłosz J. Jaguszewski ${ }^{2}$, Michael Frass ${ }^{3}$, Marcin Leskiewicz ${ }^{4}$, \\ Jacek Smereka ${ }^{4}$, Jerzy R. Ładny ${ }^{5}$, Oliver Robak ${ }^{3}$, Łukasz Szarpak ${ }^{6}$ \\ ${ }^{1}$ Polish Society of Disaster Medicine, Warsaw, Poland \\ ${ }^{2} 1^{\text {st }}$ Department of Cardiology, Medical University of Gdansk, Poland \\ ${ }^{3}$ Department of Medicine I, Medical University of Vienna, Austria \\ ${ }^{4}$ Department of Emergency Medical Service, Wroclaw Medical University, Wroclaw, Poland \\ ${ }^{5}$ Department of Emergency Medicine and Disaster, Medical University of Bialystok, Poland \\ ${ }^{6}$ Lazarski University, Warsaw, Poland
}

\begin{abstract}
Background: The aim of the study was to compare the quality of chest compressions (CCs) carried out with and without the use of the TrueCPR device during simulated cardiopulmonary resuscitations conducted by trainee doctors.

Methods: The study was a prospective, randomized, cross-over simulation study. The study involved 65 trainee doctors who were tasked with performing a 2-min cycle of uninterrupted CCs under conditions of a simulated cardiopulmonary resuscitation of adults. CC were carried out in two scenarios: with and without TrueCPR chest compression support. Participants did not have experience in the use of CCs prior to this study.

Results: The depth of compressions in regard to CC techniques were varied by $45 \mathrm{~mm}$ (IQR 43-48) for manual CC and $53 \mathrm{~mm}$ (IQR 51-55) for the TrueCPR device ( $p<0.001)$. The incidence of CCs with and without TrueCPR was: 112 (IQR 103-113) vs. 129 (IQR 122-135) compressions ( $p=0.002)$. The degree of complete chest relaxation with the TrueCPR device was 95\% (IQR 76-99) and without the device, 33\% (IQR 29-38) ( $p<0.001)$.

Conclusions: In the simulation study performed, the use of the TrueCPR device resulted in a significant improvement in the quality of CCs in relation to frequency and depth of CCs and correctness of chest relaxation. (Cardiol J 2019; 26, 5: 529-535)
\end{abstract}

Key words: cardiopulmonary resuscitation, chest compressions, quality, medical simulation, doctor

\section{Introduction}

Sudden cardiac arrest is a challenge for modern medicine, resulting not only from the scale of the phenomenon, but also the social and economic burden of the health care system [1, 2]. In Europe, it is indicated that the annual incidence of EMS-treated out-of-hospital cardiopulmonary arrests for all rhythms is 38 per 100,000 population [3].

Current guidelines for cardiopulmonary resuscitation indicate high quality chest compressions (CCs) as an element affecting the effectiveness of

Address for correspondence: Associate Professor, Łukasz Szarpak, PhD, Lazarski University, ul. Świeradowska 43, 02-662 Warszawa, Poland, e-mail: lukasz.szarpak@gmail.com

Received: 16.07.2018

Accepted: 22.07.2018 
cardiopulmonary resuscitation. The quality of CCs is made up of factors such as minimization of CCs, $\mathrm{CCs}$ to the appropriate depth and with appropriate frequency, full chest relaxation and correct positioning of the hands on the chest [4-6]. Minimizing breaks in CCs has a direct impact on increasing the perfusion pressure during resuscitation procedures, both in adults and pediatric patients [7]. As Ewy et al. [8] research indicates, the first CCs are ineffective in the context of the perfusion pressure, only compression $7-8$ is effective. This situation is repeated each time there is a break in the CCs, which includes performing rescue breaths in the case of resuscitation in a cycle of 30 compressions to 2 rescue breaths. Minimizing breaks in CCs can be achieved by instrumental protection of the airways by means of an endotracheal tube or supraglottic ventilation device and asynchronous resuscitation, during which there is no need to take breaks for rescue breaths [6, 7]. For such a solution, Ewy et al. [8] and many other authors [9, 10] point to an optimal method of resuscitation. In the case of adult $\mathrm{CCs}$, the guidelines of the European Resuscitation Council (ERC) [11], as well as the American Heart Association (AHA) [4], indicate that it should be between 50 and $60 \mathrm{~mm}$. In addition, CCs should be carried out with a frequency of 100-120 compressions per minute (cpm). Faster $\mathrm{CCs}$ may result in a higher perfusion pressure; however, a faster time causes greater fatigue to the chest compressor, which in the case of prolonged resuscitation can lead to a significant deterioration of the quality of CCs. Another important component responsible for the quality of $\mathrm{CCs}$ is the correctness of chest relaxation. Performing $\mathrm{CCs}$ at an appropriate depth and then leading to full chest relaxation results in the creation of an appropriate pressure difference in the chest, which determines the perfusion pressure. Incomplete chest relaxation will result in a reduction of perfusion pressure, thereby reducing chances of spontaneous circulation returning [12-14].

The correctness of hand position on the chest also plays an important role in the context of quality of CCs. The current guidelines $[4,5]$ recommend that the hands be placed in the middle of the chest on the sternum, in addition, the person pressing on the chest should take such a position relative to the patient that his upper limbs are at right angles to the patient's chest. Bad hand placement on the chest may cause damage to the patient's chest [15]. Performing CCs based on the above recommendations determines the highest quality of $\mathrm{CCs}$,

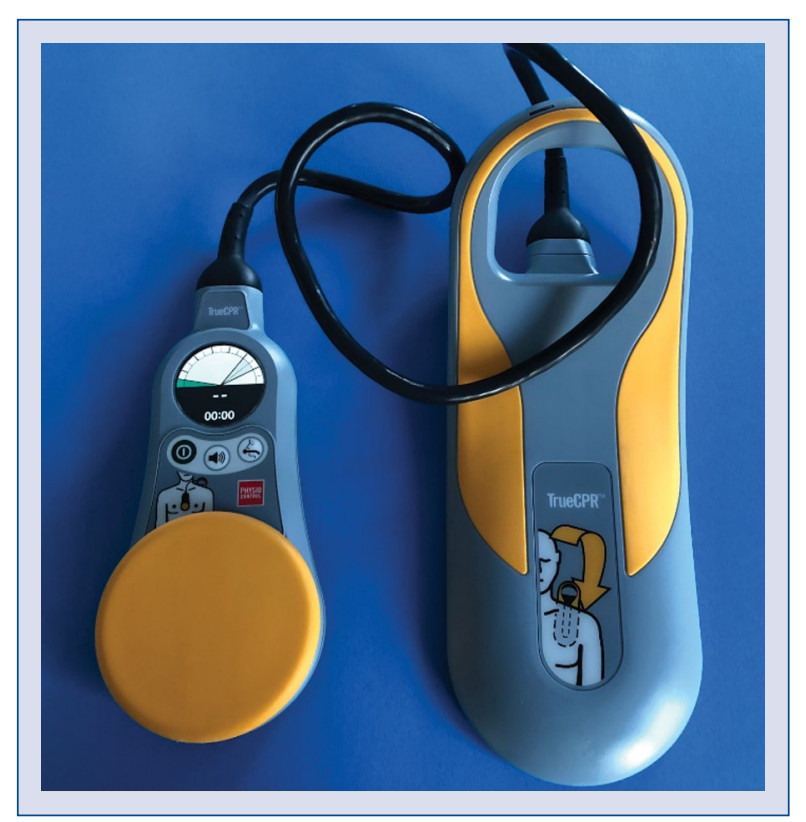

Figure 1. TrueCPR cardiopulmonary resuscitation feedback device.

translating directly to the resuscitation outcome in the form of a return of spontaneous circulation [16].

The aim of the study was to compare the quality of CCs performed unmanageaged and using the TrueCPR device (Fig. 1) during simulated cardiopulmonary resuscitation conducted by trainee doctors.

\section{Methods}

The study was a randomized, cross-over study and was conducted under conditions of medical simulation. The study protocol was accepted by the Institutional Review Board of the Polish Society of Disaster Medicine (approval no. 17/05/2017). The study was conducted from June to July 2017. The doctors qualified the trainees participating in emergency medicine courses organized by the Department of Emergency Medicine at the Medical University of Warsaw. The inclusion criteria included: completing medical studies and having the status of trainee, lack of previous experience in the use of cardiopulmonary resuscitation feedback devices, and voluntary consent to join the study.

Among the exclusion criteria were: spine injury or wrist injury during the month preceding the examination preventing $\mathrm{CCs}$, pregnancy, or non-fulfillment of inclusion criteria. Ultimately, 68 physicians were enrolled in the study, but only 


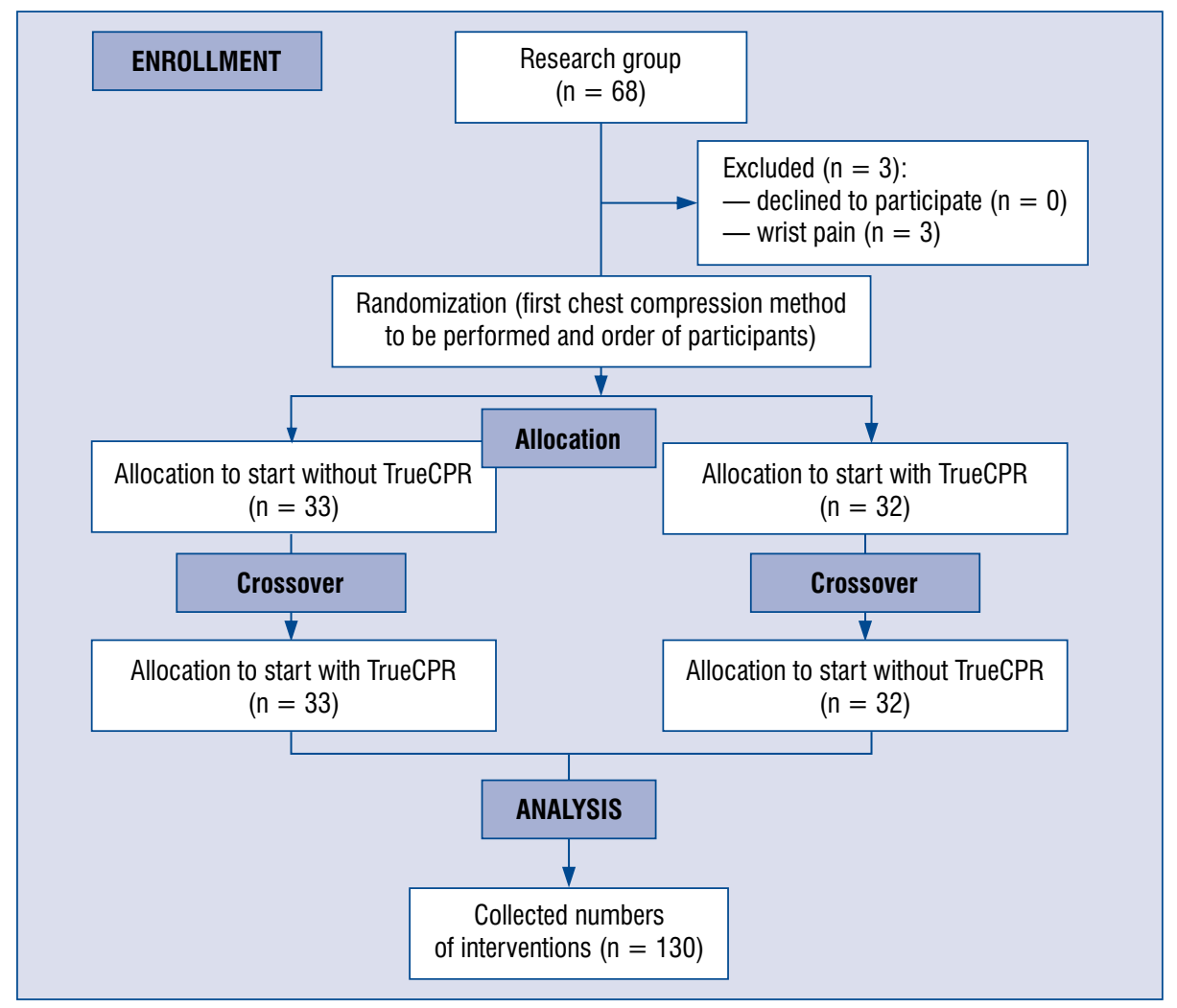

Figure 2. Randomization flow chart.

65 physicians completed the study. Three people withdrew from the study due to wrist pain (Fig. 2).

Prior to the study, all persons participated in the training module in the field of basic resuscitation procedures based on the guidelines of the American Resuscitation Council [2,3]. The correct $\mathrm{CCs}$ were shown once again in a non-instrumental manner, as well as performing cardiopulmonary resuscitation based on the TrueCPR device, which, owing to information displayed on the depth of compressions, the frequency of compressions, as well as the degree of chest relaxation, allowing for correction of quality of CCs in real time. After instruction, participants of the study had $10 \mathrm{~min}$ to read the TrueCPR device, however, practical exercises with this device were not done in advance. On the following day, participants during the target study were asked to perform CCs continuously for $2 \mathrm{~min}$ with and without the TrueCPR device. Cardiopulmonary resuscitation was carried out based on one rescuer. Both the order of the participants and the methods of CCs was random. For this purpose, the coin-toss technique was used. A detailed randomization procedure is presented on Figure 2. To simulate a patient in need of CCs, an adult simulator, Resusci Anne Simulator (Laerdal, Stavanger, Norway) was used.

During the study, only the parameters concerning the quality of CCs such as the depth of compressions, the frequency of compressions, the degree of chest relaxation and the correctness of hands on the chest during compressions were evaluated. Parameters were measured using SimPad ${ }^{\oplus}$ PLUS (Laerdal, Stavanger, Norway), which is an operating device used to control Laerdal simulators. Additionally, after completing the scenarios, the participants assessed the 100-degree scale on the level of self-confidence in the correctness of chest CCs (1 - uncertain; 100 - confident), as well as indicating which of the $\mathrm{CC}$ techniques they would prefer to use under real resuscitation conditions.

Sample size calculation was performed using $\mathrm{G}^{*}$ Power 3.1 with a two-tailed t-test (Cohen's d: 0.8 , alpha error: 0.05 , power: 0.95 ). According to the calculation, a minimum of 51 participants were necessary.

\section{Statistical analysis}

The statistical package Statistica 13.0 EN (StatSoft, Tulusa, OK, USA) was used for all sta- 
Table 1. Chest compression (CC) parameters with and without TrueCPR device.

\begin{tabular}{lccc}
\hline Parameter & Manual CC & CCs with TrueCPR & P \\
\hline CC rate $\left[\mathrm{n} \times \mathrm{min}^{-1}\right]$ & 129 [IOR 122-135] & 112 [IOR 103-113] & $\mathbf{0 . 0 0 2}$ \\
CC depth [mm] & 45 [IOR 43-48] & 53 [IOR 51-55] & $<\mathbf{0 . 0 0 1}$ \\
Full release [\%] & 33 [IOR 29-38] & 95 [IOR 76-99] & $<\mathbf{0 . 0 0 1}$ \\
Correct hand position [\%] & 94 [IOR 82-96] & 95 [IOR 89-98] & 0.185 \\
\hline
\end{tabular}

IQR - interquartile range

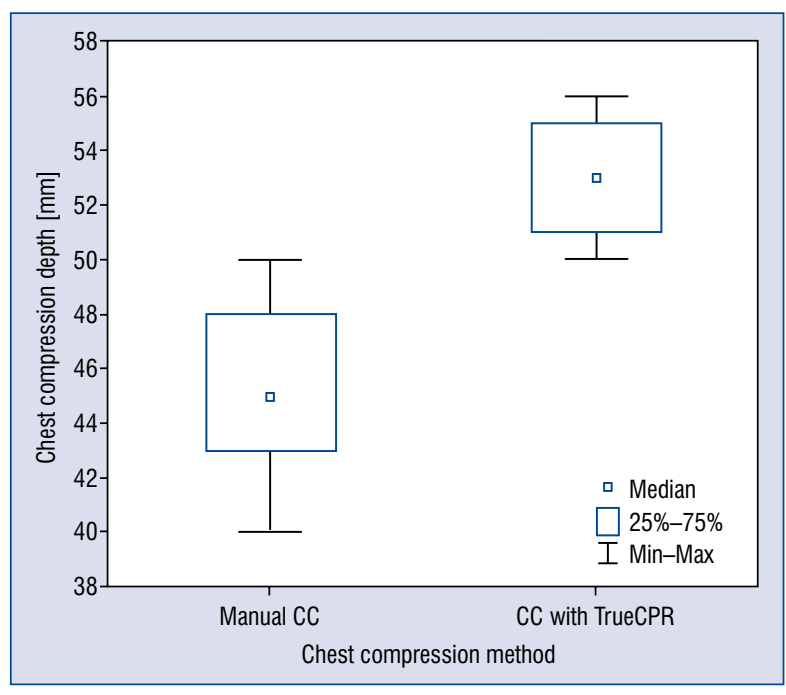

Figure 3. Median chest compression (CC) depth.

tistical analysis. Normal distribution was confirmed by the Kolmogorov-Smirnov test. When the data did not follow normal distribution, non-parametric tests were used. The participants' subjective opinions were compared with the use of the Stuart-Maxwell test. Data were presented as medians and interquartile range (IQR) or percentages (\%). The results were considered significant at $\mathrm{p}<0.05$.

\section{Results}

The study involved 65 trainee doctors $(27 \mathrm{fe}-$ male, $41.5 \%$ ), whose median age was 25 (IQR $24.5-26$ ) years. None of the participants had previous clinical or experimental experience in cardiopulmonary resuscitation feedback devices.

Detailed data on the parameters of CCs are presented in Table 1 . The depth of compressions varied in relation to the $\mathrm{CC}$ technique tested, with $45 \mathrm{~mm}$ (IQR 43-48) for manual CCs and $53 \mathrm{~mm}$ (IQR 51-55) for the TrueCPR device. This difference was statistically significant (Fig. 3, $\mathrm{p}<0.001$ ).

The frequency of CCs when the TrueCPR device was used resulted in $112 \mathrm{cpm}$ (IQR 103-113)

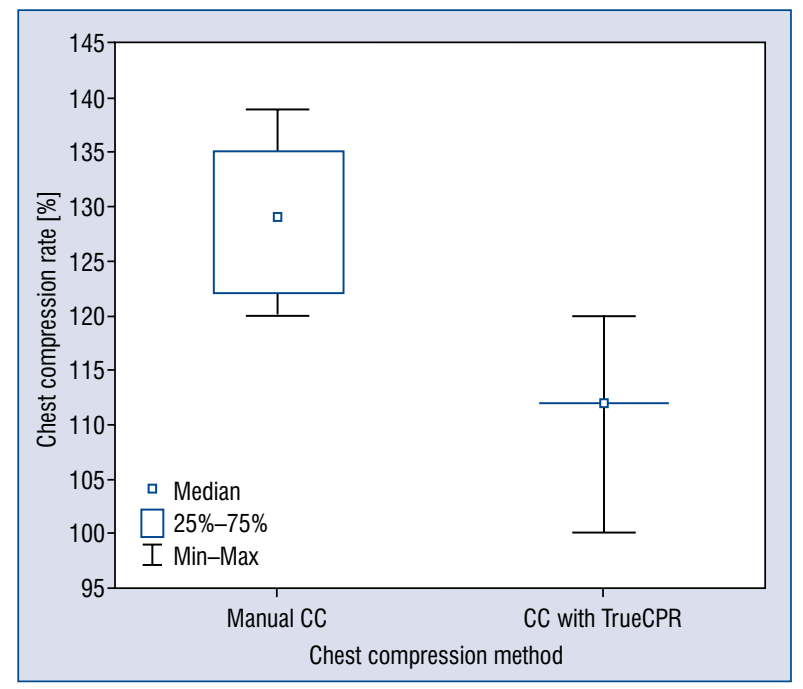

Figure 4. Median chest compression (CC) rate.

and achieved statistical significance which was lower than when not using the device during $\mathrm{CCs}$, resulting in $129 \mathrm{cpm}$ (IQR 122-135) (Fig. 4, p = 0.002).

The degree of complete chest relaxation with the TrueCPR device was 95\% (IQR 76-99) and without a device it was 33\% (IQR 29-38). The difference in the degree of correctly performed chest relaxation between the tested devices was statistically significant (Fig. 5, p < 0.001).

In the case of proper hand positioning on the chest, no statistically significant differences were observed in the examined devices (Table 1).

The degree of self-confidence of correct CCs in the absence of a device was 77 (IQR 56-87) and when using the TrueCPR device, the degree of self-confidence was higher, at 87 (IQR 73-99) $(\mathrm{p}=0.024)$.

Fifty-seven people, which accounted for $87.7 \%$ of the whole research group, declared that they would choose the TrueCPR device during routine cardiopulmonary resuscitation in their professional practice. The remaining $12.3 \%$ of people opted for the device-less CCs as their preferred method of cardiopulmonary resuscitation. 


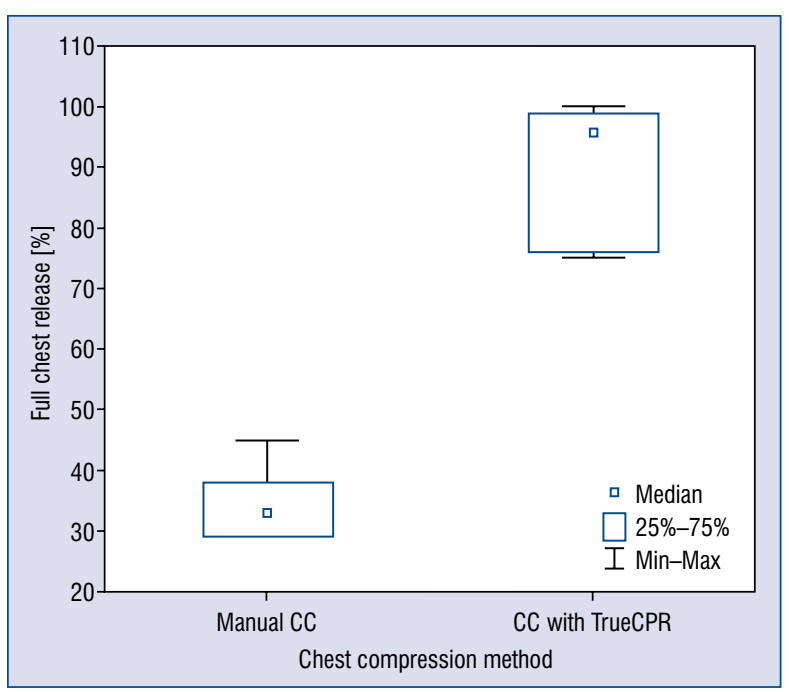

Figure 5. Degree of chest compression (CC) relaxation.

\section{Discussion}

In this simulation study, the impact of using the cardiopulmonary resuscitation feedback device versus TrueCPR on the quality of CCs performed by trainee doctors was assessed. The results obtained indicate an advantage of the method of using the device over not using the device in CCs.

Cardiopulmonary resuscitation is one of the most stressful situations that a physician can encounter during their daily work experience [17]. It is dictated by the necessity of implementing advanced resuscitation activities and to conduct them until the arrival of an emergency medical team or the arrival of a resuscitation team [18].

In the present study, a statistically significant difference was found between the depth of $\mathrm{CCs}$ with and the depth without the TrueCPR device (53 vs. $45 \mathrm{~mm}$, respectively). Current guidelines for cardiopulmonary resuscitation recommends that the depth of CCs in adults is between 50 and $60 \mathrm{~mm}$ [4]. As shown by studies conducted by Lampe et al. [19], deeper CCs redirect several hemodynamic parameters. Iskrzycki et al. [20] conducting studies on the effectiveness of cardiopulmonary resuscitation carried out by water rescuers, demonstrated that the use of visual real-time feedback devices has improved the quality of cardiopulmonary resuscitation in relatively unexperienced cardiopulmonary resuscitation providers. In the case of prolonged resuscitation, when the person performing CCs becomes fatigued, the use of feedback devices that are able to correct the quality of CCs is all the more justified. Buléon et al. [21] indicated that the real-time feedback device delivers longer, effective, and steadier CCs over time. Other authors have also come to similar conclusions [4, 6, 22-24].

Another important factor influencing the return of spontaneous circulation is the frequency of CCs [16]. Field et al. [25] showed that a CC rate of $100-120 / \mathrm{min}^{-1}$ for $2 \mathrm{~min}$ is feasible whilst maintaining adequate $\mathrm{CC}$ quality in terms of depth, duty-cycle, leaning, and decay in compression performance. These are the parameters currently recommended by guidelines for cardiopulmonary resuscitation [4]. In turn, studies by Lee et al. [26] showed that the frequency of compressions over $120 /$ min was associated with a higher depth of compressions than the frequency of compressions indicated in the guidelines for resuscitation [4]. Zou et al. [27] indicated the most optimal frequency of CCs is a frequency of $120 / \mathrm{min}$ [26, 27]. In the present study, the compression rate performed without the device by trainee doctors was $129 \mathrm{cpm}$, while in the case of using the TrueCPR device it was $112 \mathrm{cpm}$. The reduction in frequency using cardiopulmonary resuscitation feedback devices may be dictated by the fact that these devices (including TrueCPR) display the current pressure of the compressions performed by the rescuer, thanks to which they are able to adapt to the frequency recommended by the resuscitation guidelines. Wee et al. [28] indicate that the use of feedback devices helps improve the quality of cardiopulmonary resuscitation during training. This opinion is also shared by other authors dealing with the issue of improving the effectiveness of resuscitation [20, 29-32].

Full chest relaxation after each compression also plays a significant role in the quality of cardiopulmonary resuscitation and the frequency of return of spontaneous circulation and is one of the recommendations of the AHA as well as the ERC $[4,11]$. Luire points out that complete chest wall recoil improves hemodynamics during cardiopulmonary resuscitation by generating relatively negative intrathoracic pressure, which draws venous blood back to the heart, and provides cardiac preload prior to the next CC [33]. In the study, the intern doctors tended to perform full chest relaxation just to the recommended level. Thanks to the use of the feedback device, this percentage increased to 95\%. The TrueCPR device, due to the scales on the display, indicates whether the person performing CCs fully relaxes it, in regards to the frequency and depth of compressions, making it possible to correct these parameters if needed. $\mathrm{Nu}-$ merous studies comparing CCs with compressions 
using feedback devices or mechanical CC devices indicate that a tendency towards incomplete chest relaxation during resuscitation concerns not only physicians but other professional groups as well $[12,28,34,35]$.

In the study conducted, the participants indicated that their confidence in the correctness of $\mathrm{CCs}$ increased in the case of using a cardiopulmonary resuscitation feedback device. This may be related to the fact that the device, so to say, tells people performing $\mathrm{CCs}$ what parameters to pay attention to and how to improve them to achieve optimal quality of performed procedures.

\section{Limitations of the study}

The study has specific limitations resulting from the methodology. Firstly, the study was conducted under simulated conditions, not real cardiopulmonary resuscitation, however, this choice was intentional, because the medical simulation allows for full standardization of conditions for procedures performed, moreover, it was the only one that allowed cross-randomized trials without potential damage to a patient's health [36-39]. Additionally, thanks to the use of advanced simulators, it was possible to obtain the data regarding quality of CCs performed without an accompanying feedback device. The second limitation was to perform CCs in a 2-min cycle, however, such a period of $\mathrm{CCs}$ is recommended by the guidelines for resuscitation $[13,39,40]$ and after this time there should be a change in the person performing CCs. The strength of the study is its randomized, cross-over nature, and the use of one of the most advanced feedback devices available

\section{Conclusions}

In the simulation test conducted, the use of the TrueCPR device resulted in a significant improvement in the quality of $\mathrm{CCs}$ in relation to the frequency and depth of CCs and the correctness of chest relaxation performed by trainee doctors.

\section{References}

1. Kempa M, Królak T, Drelich $~ €$, et al. Pre-discharge defibrillation testing: Is it still justified? Cardiol J. 2016; 23(5): 532-538, doi: 10.5603/CJ.a2016.0045, indexed in Pubmed: 27387062.

2. Freund B, Kaplan PW. A review of the utility of a hypothermia protocol in cardiac arrests due to non-shockable rhythms. Cardiol J. 2017; 24(3): 324-333, doi: 10.5603/CJ.a2017.0016, indexed in Pubmed: 28150290.

3. Monsieurs KG, Nolan JP, Bossaert LL, et al. European Resuscitation Council Guidelines for Resuscitation 2015: Section 1. Ex- ecutive summary. Resuscitation. 2015; 95: 1-80, doi: 10.1016/j. resuscitation.2015.07.038, indexed in Pubmed: 26477410.

4. Link MS, Berkow LC, Kudenchuk PJ, et al. Part 7: Adult Advanced Cardiovascular Life Support: 2015 American Heart Association Guidelines Update for Cardiopulmonary Resuscitation and Emergency Cardiovascular Care. Circulation. 2015; 132(18 Suppl 2): S444-S464, doi: 10.1161/CIR.0000000000000261, indexed in Pubmed: 26472995.

5. Kleinman ME, Brennan EE, Goldberger ZD, et al. Part 5: Adult Basic Life Support and Cardiopulmonary Resuscitation Quality: 2015 American Heart Association Guidelines Update for Cardiopulmonary Resuscitation and Emergency Cardiovascular Care. Circulation. 2015; 132(18 Suppl 2): S414-S435, doi: 10.1161/ CIR.0000000000000259, indexed in Pubmed: 26472993.

6. Kurowski A, Szarpak $€$, Bogdański $€$, et al. Comparison of the effectiveness of cardiopulmonary resuscitation with standard manual chest compressions and the use of TrueCPR and PocketCPR feedback devices. Kardiol Pol. 2015; 73(10): 924-930, doi: 10.5603/KP.a2015.0084, indexed in Pubmed: 25985725.

7. Zideman DA, De Buck EDJ, Singletary EM, et al. European Resuscitation Council Guidelines for Resuscitation 2015 Section 9. First aid. Resuscitation. 2015; 95: 278-287, doi: 10.1016/j.resuscitation.2015.07.031, indexed in Pubmed: 26477417.

8. Ewy GA, Zuercher M, Hilwig RW, et al. Improved neurological outcome with continuous chest compressions compared with 30:2 compressions-to-ventilations cardiopulmonary resuscitation in a realistic swine model of out-of-hospital cardiac arrest. Circulation. 2007; 116(22): 2525-2530, doi: 10.1161/CIRCULATIONAHA.107.711820, indexed in Pubmed: 17998457.

9. Nishiyama C, Iwami T, Kawamura T, et al. Quality of chest compressions during continuous CPR; comparison between chest compression-only CPR and conventional CPR. Resuscitation. 2010; 81(9): 1152-1155, doi: 10.1016/j.resuscitation.2010.05.008, indexed in Pubmed: 20732607.

10. Chalkias A, Iacovidou N, Xanthos T. Continuous chest compression pediatric cardiopulmonary resuscitation after witnessed electrocution. Am J Emerg Med. 2014; 32(6): 686.e1-686.e2, doi: 10.1016/j.ajem.2013.12.003, indexed in Pubmed: 24418444.

11. Neumar RW, Shuster M, Callaway CW, et al. Part 1: Executive Summary: 2015 American Heart Association Guidelines Update for Cardiopulmonary Resuscitation and Emergency Cardiovascular Care. Circulation. 2015; 132(18 Suppl 2): S315S367, doi: 10.1161/CIR.0000000000000252, indexed in Pubmed: 26472989.

12. Smereka J, Szarpak L, Rodríguez-Núñez A, et al. A randomized comparison of three chest compression techniques and associated hemodynamic effect during infant CPR: A randomized manikin study. Am J Emerg Med. 2017; 35(10): 1420-1425, doi: 10.1016/j.ajem.2017.04.024, indexed in Pubmed: 28433454.

13. Smereka J, Kasiński M, Smereka A, et al. The quality of a newly developed infant chest compression method applied by paramedics: a randomised crossover manikin trial. Kardiol Pol. 2017; 75(6): 589-595, doi: 10.5603/KP.a2017.0015, indexed in Pubmed: 28150278 .

14. Treptau J, Ebnet J, Akin M, et al. Angiographic detection of fatal acute aortic dissection Stanford type A under resuscitation. Cardiol J. 2016; 23(6): 620-622, doi: 10.5603/CJ.2016.0103, indexed in Pubmed: 27976792.

15. Kim MJ, Park YS, Kim SW, et al. Chest injury following cardiopulmonary resuscitation: a prospective computed tomography evaluation. Resuscitation. 2013; 84(3): 361-364, doi: 10.1016/j. resuscitation.2012.07.011, indexed in Pubmed: 22819881. 
16. Kurowski A, Czyżewski L, Bogdański L, et al. Quality of chest compression with CardioPump CPR compared to single rescuer standard BLS. Am J Emerg Med. 2015; 33(1): 114-115, doi: 10.1016/j.ajem.2014.10.027, indexed in Pubmed: 25455057.

17. Szarpak L, Filipiak KJ, Ładny JR, et al. Should nurses use mechanical chest compression devices during CPR? Am J Emerg Med. 2016; 34(10): 2044-2045, doi: 10.1016/j.ajem.2016.07.057, indexed in Pubmed: 27528048.

18. Szarpak $€$, Truszewski $Z$, Smereka J, et al. Does the use of a chest compression system in children improve the effectiveness of chest compressions? A randomised crossover simulation pilot study. Kardiol Pol. 2016; 74(12): 1499-1504, doi: 10.5603/ KP.a2016.0107, indexed in Pubmed: 27391911.

19. Lampe JW, Tai Y, Bratinov G, et al. Developing a kinematic understanding of chest compressions: the impact of depth and release time on blood flow during cardiopulmonary resuscitation. Biomed Eng Online. 2015; 14: 102, doi: 10.1186/s12938-0150095-4, indexed in Pubmed: 26537881.

20. Iskrzycki L, Smereka J, Rodriguez-Nunez A, et al. The impact of the use of a CPRMeter monitor on quality of chest compressions: a prospective randomised trial, cross-simulation. Kardiol Pol. 2018; 76(3): 574-579, doi: 10.5603/KP.a2017.0255, indexed in Pubmed: 29297195.

21. Buléon C, Delaunay J, Parienti JJ, et al. Impact of a feedback device on chest compression quality during extended manikin CPR: a randomized crossover study. Am J Emerg Med. 2016; 34(9): 1754-1760, doi: 10.1016/j.ajem.2016.05.077, indexed in Pubmed: 27349359.

22. Truszewski Z, Szarpak L, Kurowski A, et al. Randomized trial of the chest compressions effectiveness comparing 3 feedback CPR devices and standard basic life support by nurses. Am J Emerg Med. 2016; 34(3): 381-385, doi: 10.1016/j.ajem.2015.11.003, indexed in Pubmed: 26612703.

23. Abelairas-Gómez C, Barcala-Furelos R, Szarpak $€$, et al. The effect of strength training on quality of prolonged basic cardiopulmonary resuscitation. Kardiol Pol. 2017; 75(1): 21-27, doi: 10.5603/KP.a2016.0165, indexed in Pubmed: 27878801.

24. Smereka J, Szarpak L, Smereka A, et al. Evaluation of new two-thumb chest compression technique for infant CPR performed by novice physicians. A randomized, crossover, manikin trial. Am J Emerg Med. 2017; 35(4): 604-609, doi: 10.1016/j. ajem.2016.12.045, indexed in Pubmed: 28040386.

25. Field RA, Soar J, Davies RP, et al. The impact of chest compression rates on quality of chest compressions: a manikin study. Resuscitation. 2012; 83(3): 360-364, doi: 10.1016/j.resuscitation.2011.07.012, indexed in Pubmed: 21771570.

26. Lee SH, Kim K, Lee JH, et al. Does the quality of chest compressions deteriorate when the chest compression rate is above 120/min? Emerg Med J. 2014; 31(8): 645-648, doi: 10.1136/ emermed-2013-202682, indexed in Pubmed: 23704754.

27. Zou Y, Shi W, Zhu Y, et al. Rate at 120 /min provides qualified chest compression during cardiopulmonary resuscitation. Am J Emerg Med. 2015; 33(4): 535-538, doi: 10.1016/j.ajem.2015.01.024, indexed in Pubmed: 25662803.

28. Wee JCP, Nandakumar M, Chan YH, et al. Effect of using an audiovisual CPR feedback device on chest compression rate and depth. Ann Acad Med Singapore. 2014; 43(1): 33-38, indexed in Pubmed: 24557463.
29. Krasteva V, Jekova I, Didon JP. An audiovisual feedback device for compression depth, rate and complete chest recoil can improve the CPR performance of lay persons during self-training on a manikin. Physiol Meas. 2011;32(6): 687-699, doi: 10.1088/09673334/32/6/006, indexed in Pubmed: 21606561.

30. Ruiz de Gauna S, González-Otero DM, Ruiz J, et al. Feedback on the rate and depth of chest compressions during cardiopulmonary resuscitation using only accelerometers. PLoS One. 2016; 11(3): e0150139, doi: 10.1371/journal.pone.0150139, indexed in Pubmed: 26930061.

31. Wieczorek W, Smereka J, Ladny JR, et al. The impact of a CPRezy ${ }^{\mathrm{TM}}$ feedback device on the quality of chest compressions performed by nurses. Am J Emerg Med. 2018; 36(7): 1318-1319, doi: 10.1016/j.ajem.2017.11.061, indexed in Pubmed: 29196113.

32. Kirkbright S, Finn J, Tohira H, et al. Audiovisual feedback device use by health care professionals during CPR: a systematic review and meta-analysis of randomised and non-randomised trials. Resuscitation. 2014; 85(4): 460-471, doi: 10.1016/j.resuscitation.2013.12.012, indexed in Pubmed: 24361457.

33. Lurie K, Voelckel W, Plaisance P, et al. Use of an inspiratory impedance threshold valve during cardiopulmonary resuscitation: a progress report. Resuscitation. 2000; 44(3): 219-230, indexed in Pubmed: 10825624.

34. Wieczorek W, Kaminska H. Impact of a corpuls CPR Mechanical Chest Compression Device on chest compression quality during extended pediatric manikin resuscitation: a randomized crossover pilot study. Disaster Emerg Med J. 2017; 2(2): 58-63, doi: 10.5603/DEMJ.2017.0012.

35. Beckers SK, Skorning MH, Fries M, et al. CPREzy improves performance of external chest compressions in simulated cardiac arrest. Resuscitation. 2007; 72(1): 100-107, doi: 10.1016/j.resuscitation.2006.05.020, indexed in Pubmed: 17079067.

36. Szarpak L, Truszewski Z, Czyzewski L, et al. A comparison of the McGrath-MAC and Macintosh laryngoscopes for child tracheal intubation during resuscitation by paramedics. A randomized, crossover, manikin study. Am J Emerg Med. 2016; 34(8): 1338-1341, doi: 10.1016/j.ajem.2015.11.060, indexed in Pubmed: 26712571.

37. Szarpak $€$, Czyżewski $Ł$, Kurowski A. Comparison of the Pentax, Truview, GlideScope, and the Miller laryngoscope for child intubation during resuscitation. Am J Emerg Med. 2015; 33(3): 391-395, doi: 10.1016/j.ajem.2014.12.020, indexed in Pubmed: 25662205.

38. Evrin T, Bielski KT. Is there any difference between different infant chest compression methods? Disaster Emerg Med J. 2017; 2(4): 173-174, doi: 10.5603/DEMJ.2017.0039.

39. Frass M, Robak O, Truszewski $Z$, et al. Comparison of endotracheal intubation with the airtraq avant ${ }^{\circledR}$ and the macintosh laryngoscope during intermittent or continuous chest compression: a randomized, crossover study in manikins. Disaster Emerg Med J. 2016; 1(1): 7-13, doi: 10.5603/DEMJ.2016.0002.

40. Aleksandrowicz S, Madziala M, Iskrzycki L, et al. Performance of chest compressions with the use of the new mechanical chest compression machine lifeline arm: a randomized crossover manikin study in novice physicians. Disaster Emerg Med J. 2016; 1(1): 30-36, doi: 10.5603/DEMJ.2016.0005. 